\title{
OPTIMAL FINGERPRINTING STRATEGIES WITH ONE-SIDED ERROR
}

\author{
A.J. SCOTT ${ }^{a}$ JONATHAN WALGATE BARRY C. SANDERS \\ Institute for Quantum Information Science, University of Calgary \\ Calgary, Alberta T2N 1N4, Canada
}

Received November 29, 2005

Revised July 6, 2006

\begin{abstract}
Fingerprinting enables two parties to infer whether the messages they hold are the same or different when the cost of communication is high: each message is associated with a smaller fingerprint and comparisons between messages are made in terms of their fingerprints alone. In the simultaneous message passing model, it is known that fingerprints composed of quantum information can be made exponentially smaller than those composed of classical information. For small message lengths, we present constructions of optimal classical fingerprinting strategies with one-sided error, in both the one-way and simultaneous message passing models, and provide bounds on the worst-case error probability with the help of extremal set theory. The performance of these protocols is then compared to that for quantum fingerprinting strategies constructed from spherical codes, equiangular tight frames and mutually unbiased bases.
\end{abstract}

Keywords: quantum fingerprinting, communication complexity

Communicated by: I Cirac \& M Mosca

\section{Introduction}

Consider the following scenario. Alice needs to send an important message to Bob. She does not mind if an intermediary party reads this message while in transit, but does however, require that the message remains unchanged. Alice considers broadcasting her message through a trusted public communication channel, her local radio station, but alas, access time is very expensive and her message is long. Instead she proceeds by sending it across untrusted communication channels, over the internet, which are inexpensive and plentiful. Alice is now faced, however, with the possibility that a saboteur has surreptitiously intercepted and changed part, or all, of her message. To test its integrity, she reluctantly reconsiders buying airtime at her local radio station. Alice's transmission costs now depend on the level of risk she is prepared to tolerate, for a corrupted message to pass undetected. An absolute guarantee of integrity requires Alice to transmit her entire message again, this time through the trusted channel. If however, she is willing to tolerate a small probability of detection error, a considerably smaller piece of information, called a fingerprint, need only be sent. Alice could, for example, broadcast only the first byte of her message. This strategy is said to have one-sided error: when the first byte does not match that for Bob's copy, Alice knows with certainty that foul play has occurred. Unfortunately, since it is Bob who must perform the

$a_{\text {email: ascott@qis.ucalgary.ca }}$ 
comparison, and no private communication channel is available, we are forced to assume that Alice and Bob use a strategy which is publicly known. An adversary will now simply avoid corrupting the first byte, while playing havoc with the rest. There is, of course, a simple workaround: Alice instead broadcasts a random byte of her message, chosen by flipping a private coin, together with the location of this byte. Assuming the availability of a trusted, though costly, broadcast channel, Alice has thus obtained a level of security through only public communication. In a probabilistic sense, message sabotage will never go undetected.

Although motivated in terms of message authentication, the preceding protocol is called fingerprinting. In all generality, fingerprinting enables separated parties to infer whether the messages they hold are the same or different when the cost of communication is high: each message is associated with a smaller fingerprint, and comparisons between messages are made in terms of their fingerprints alone. In the above authentication scheme, the messages were compared using one-way communication, from Alice to Bob. It is known from the theory of communication complexity $[1,2]$, that in the limit of long messages, it is sufficient, and necessary, for Alice to communicate fingerprints of length $\Theta(\log N)$ bits, for messages of length $N$ bits, if a small constant error probability is allowed. This error probability can then be made arbitrarily small through repeated use of the protocol. In another model of communication complexity, called the simultaneous message passing (SMP) model [2], the fingerprints are transmitted by both Alice and Bob, but this time to a referee, who decides the outcome of the message comparison. In this case it is sufficient, and necessary, for Alice and Bob to communicate fingerprints of length $\Theta(\sqrt{N})$ bits if the error is to be kept arbitrarily small $[3,4,5]$.

We will study both communication models by considering the following generalized scenario. A supplier, who we call Sapna, chooses two messages, $x$ and $y$, from a pool of $n$ unique messages and hands them to Alice and Bob, respectively. Alice and Bob are now tasked with determining whether the messages they hold are the same or different. They are forbidden direct communication, however, but instead allowed to correspond with a referee, called Roger. As communication is considered expensive, Alice and Bob are limited to sending fingerprints of their original messages, $a$ and $b$ respectively, which they select from smaller pools of size $m_{A}$ and $m_{B}$. Roger then infers

$$
\operatorname{EQ}(x, y)= \begin{cases}1, & \text { if } x=y \\ 0, & \text { if } x \neq y\end{cases}
$$

and completes the protocol by announcing a single bit $z \in\{0,1\}$. Roger is correct if $z=$ $\mathrm{EQ}(x, y)$. In the current investigation we are concerned only with one-sided-error protocols, in which case, $z=0$ is allowed only if $x \neq y$. Then, when Roger announces 0, Alice and Bob can conclusively claim that the messages are indeed different. Protocols with one-sided error are of vital practical importance whenever the cost of false negative results outweighs that of false positives. This is the case when $x$ and $y$ are in fact expected to be equal. In the above authentication scheme, the one-sided-error condition means that Bob will always accept an uncorrupted message as authentic, and thus, transmission throughput remains unaffected when an adversary is not present.

The fingerprinting protocol adopted by Alice, Bob and Roger is publicly announced. The goal of this protocol is to minimize Roger's error probability. Sapna, however, may be a saboteur, and always choose message pairs that lead to the highest error rate in Roger's 
output. We thus evaluate fingerprinting protocols according to this worst-case scenario. The worst-case error probability, $P_{\text {wce }}=\max _{x, y} \operatorname{Prob}(z \neq \mathrm{EQ}(x, y))$, then corresponds to the maximum error rate, and provides an absolute guarantee on the performance of the protocol.

Note that the one-way communication model is realized when $m_{B}=n$, in which case Bob has the same number of fingerprints as Sapna has messages, and may simply pass on $y$ to Roger. Bob and Roger may now be thought of as the same party. The simultaneous message passing model is realized when $m_{A}=m_{B}$. We are concerned with the small message limit, however, where it makes more sense to analyze both models within the above general scenario. One could also consider a model where two-way communication between Alice and Bob is allowed. The added possibility of multiple communication rounds, however, would make the analysis of this model considerably more complex. We thus exclude this case.

Quantum fingerprinting protocols $[6,7,8,9]$ allow each classical fingerprint to be replaced by a quantum state. For a fair comparison, the quantum fingerprints are drawn from a Hilbert space of dimension equal to the number of available classical fingerprints. That is, instead of drawing from sets of size $m_{A}$ and $m_{B}$, respectively, Alice and Bob draw their quantum fingerprints from Hilbert spaces of dimension $m_{A}$ and $m_{B}$. In the simultaneous message passing model it is known that fingerprints composed of quantum information can be made exponentially smaller than those composed of classical information. Specifically, for messages of length $N$ bits, it was shown by Buhrman et al. [6] that fingerprints composed of $\Theta(\log N)$ qubits are sufficient, and necessary, to keep the error arbitrarily small. This exponential resource advantage is not apparent in the one-way communication model, where the classical and quantum complexities are equal.

In the current article we will investigate optimal fingerprinting strategies in the small message limit. We will present specific constructions of classical fingerprinting strategies which derive from constant-weight codes, and provide lower bounds on the worst-case error probability with the help of extremal set theory. These bounds define error rates which quantum protocols must surpass in order to claim a definitive advantage over classical protocols, and are thus important for current experimental tests of quantum fingerprinting [10,11]. In most cases the aforementioned classical fingerprinting strategies are optimal, having error rates that meet a lower bound. Our results for classical fingerprinting will be contained in Sec. 2, which is further subdivided into a preliminary discussion to set notation, an indepth study of fingerprinting under the one-way communication model, and a study of general fingerprinting which concentrates on the SMP model. In Sec. 3 we will investigate the extent at which classical strategies are outperformed by quantum fingerprinting strategies. Constructions of quantum strategies will be presented which derive from spherical codes, equiangular tight frames and mutually unbiased bases. Finally, in Sec. 4 we summarize our results.

\section{Classical fingerprinting}

\subsection{Notation and preliminary results}

It will prove useful to think of Alice, Bob and Roger forming a team with the common goal of minimizing Roger's error probability, and Sapna operating as their opponent. This team uses a pre-established, publicly known protocol. In this protocol, Alice and Bob have a fixed probability of communicating each fingerprint pair $(a, b)$ to Roger for given message pair $(x, y)$ provided by Sapna. Furthermore, Roger has a fixed probability of declaring $x$ and $y$ to be 
the same message upon receipt of fingerprint pair $(a, b)$.

Any classical fingerprinting protocol is completely specified by three functions: $p:\{1, \ldots$, $\left.m_{A}\right\} \times\{1, \ldots, n\} \rightarrow[0,1], q:\left\{1, \ldots, m_{B}\right\} \times\{1, \ldots, n\} \rightarrow[0,1]$ and $r:\left\{1, \ldots, m_{A}\right\} \times$ $\left\{1, \ldots, m_{B}\right\} \rightarrow[0,1]$. The function $p(a \mid x)$ is the probability that Alice sends fingerprint $a$ to Roger, given that she receives message $x$ from Sapna. Similarly, $q(b \mid y)$ is the probability that Bob sends $b$ to Roger, given that he receives $y$ from Sapna. The function $r(a, b)$ is the probability that Roger outputs $z=1$, given that he receives fingerprint $a$ from Alice and $b$ from Bob.

We will call the triple $(p, q, r)$ a strategy. When a party's private strategy (i.e. $p, q$ or $r$ ) takes values only in the set $\{0,1\}$, we call that party's strategy deterministic. If all parties' strategies are deterministic we call the triple $(p, q, r)$ a deterministic strategy. Otherwise a general (i.e. probabilistic) strategy should be assumed. Normalization requires

$$
\sum_{a=1}^{m_{A}} p(a \mid x)=\sum_{b=1}^{m_{B}} q(b \mid y)=1
$$

for all $x$ and $y$.

Given a strategy $(p, q, r)$, the probability that Roger outputs 1 when Sapna deals $x$ to Alice and $y$ to Bob is

$$
P_{1}^{(p, q, r)}(x, y) \equiv \sum_{a, b} p(a \mid x) q(b \mid y) r(a, b) .
$$

A strategy is said to have one-sided error when

$$
P_{1}^{(p, q, r)}(x, x)=1
$$

for all $x$. Using such a strategy, it is impossible for Roger to announce 0 when Sapna has supplied Alice and Bob with identical messages.

Defining the error probability

$$
P_{\mathrm{e}}^{(p, q, r)}(x, y) \equiv\left\{\begin{array}{ll}
1-P_{1}^{(p, q, r)}(x, x), & x=y \\
P_{1}^{(p, q, r)}(x, y), & x \neq y
\end{array},\right.
$$

the average error probability is then

$$
P_{\text {ave }}^{(p, q, r)} \equiv \frac{1}{n^{2}} \sum_{x, y} P_{\mathrm{e}}^{(p, q, r)}(x, y),
$$

and the minimum achievable average error probability is

$$
\mathcal{P}_{\text {ave }}\left(n, m_{A}, m_{B}\right) \equiv \min _{p, q, r} P_{\text {ave }}^{(p, q, r)},
$$

where the minimum is taken over all strategies. If, however, the minimum is restricted to only those strategies with one-sided error, we use $\mathcal{P}_{\text {ave }}^{1}\left(n, m_{A}, m_{B}\right)$ to denote the corresponding minimum achievable average error probability. It is this second quantity which will be of interest in the current article. The results of Horn et al. [12] immediately give us our first result: 


\section{Theorem 1.}

$$
\mathcal{P}_{\text {ave }}^{1}\left(n, m_{A}, m_{B}\right)=\frac{k\lceil n / m\rceil^{2}+(m-k)\lfloor n / m\rfloor^{2}-n}{n^{2}}
$$

where $m=\min \left\{m_{A}, m_{B}\right\}$ and $k=n \bmod m$.

When $m_{A}=m_{B}=m$ the proof of Theorem 1 follows immediately from Lemmas 2 and 3 of [12]. The last paragraph in Sec. 2 of [12] explains how to deal with the general case. The strategy which achieves the optimal error probability, given by Eq. (20) of [12], is in fact deterministic.

Although the average error probability is a perfectly legitimate measure of the performance of a fingerprinting strategy, we will consider the worst-case error probability in this article, which corresponds to the guaranteed performance of a strategy. The worst-case error probability is simply the largest error probability that Sapna can coerce:

$$
P_{\mathrm{wce}}^{(p, q, r)} \equiv \max _{x, y} P_{\mathrm{e}}^{(p, q, r)}(x, y) .
$$

Similar to the above, we define the minimum achievable worst-case error probability

$$
\mathcal{P}_{\text {wce }}\left(n, m_{A}, m_{B}\right) \equiv \min _{p, q, r} P_{\mathrm{wce}}^{(p, q, r)}=\min _{p, q, r} \max _{x, y} P_{\mathrm{e}}^{(p, q, r)}(x, y),
$$

and denote by $\mathcal{P}_{\text {wce }}^{1}\left(n, m_{A}, m_{B}\right)$ the minimum achievable worst-case error probability when restricted to one-sided-error strategies. Our objective in the following sections is to find bounds on this second quantity.

To begin, let us introduce a lemma that allows the following simplification. Whereas Roger can use a probabilistic strategy, we show that there exists a deterministic strategy for Roger, that is at least as good as all probabilistic strategies.

Lemma 2. Let $(p, q, r)$ be a fingerprinting strategy with one-sided error. Then

$$
P_{\mathrm{e}}^{(p, q, r)}(x, y) \geq P_{\mathrm{e}}^{\left(p, q, r^{\prime}\right)}(x, y)
$$

for all $x$ and $y$, where

$$
r^{\prime}(a, b)=\left\{\begin{array}{ll}
1, & \text { if } p(a \mid x)>0 \text { and } q(b \mid x)>0 \text { for some } x \\
0, & \text { otherwise }
\end{array} .\right.
$$

The proof of this lemma is straightforward and follows from enforcing the one-sided-error constraint [Eq. (4)], by setting $r^{\prime}(a, b)=1$ for each appropriate fingerprint pair $(a, b)$, and then choosing $r^{\prime}(a, b)=0$ in the remaining cases for optimality [12]. We define a binary strategy $(p, q, r)$ to be one where $r \equiv r^{\prime}$, as determined by Eq. (12). All binary strategies have one-sided error, and due to Lemma 2, our search for optimal one-sided-error strategies will be limited to this type. Each particular binary matrix $r(a, b)$ can be thought of as defining a class of possible strategies for Alice and Bob, i.e. those strategies $p$ and $q$ which do not contradict Eq. (12). In the following sections we will decide which choices for $r(a, b)$ are best.

\subsection{One-way model: $m_{B}=n$}

The simplest non-trivial examples of classical fingerprinting occur when $m_{B}=n$, in which case Bob may simply pass on $y$ to Roger. In general, when $m_{B}=n$ we will always set 
$m_{A}=m, q(b \mid y)=\delta_{b y}$, and then refer to the strategy only by the pair $(p, r)$. A binary strategy is then one with

$$
r(a, x)=\left\{\begin{array}{ll}
1, & \text { if } p(a \mid x)>0 \\
0, & \text { otherwise }
\end{array},\right.
$$

and thus, Alice's decisions will determine Roger's. Alternatively, we may think of Roger's binary decision matrix, $r(a, x)$, as specifying a class of strategies with $p(a \mid x)>0$ whenever $r(a, x)=1$, and 0 otherwise. Our job is to then minimize the worst-case error probability

$$
P_{\mathrm{wce}}^{(p, r)}=\max _{x \neq y} P_{1}^{(p, r)}(x, y)
$$

over all such members of this class, where

$$
P_{1}^{(p, r)}(x, y)=\sum_{a=1}^{m} p(a \mid x) r(a, y) .
$$

We first investigate cases where the values of $m$ and $n$ restrict all strategies to those with $P_{\mathrm{wce}}^{(p, r)}=1$. First note that given $\sum_{a} p(a \mid x)=1$ and $r(a, y) \leq 1$, we will have $P_{1}(x, y)=1$ if and only if $r(a, y)=1$ whenever $p(a \mid x)>0$, or, for binary strategies, $r(a, y)=1$ whenever $r(a, x)=1$. Defining index sets for Alice's fingerprinting strategy

$$
I_{x}^{(p)} \equiv\{a \mid p(a \mid x)>0\}
$$

which specify Roger's decisions

$$
r(a, x)=\left\{\begin{array}{ll}
1, & \text { if } a \in I_{x}^{(p)} \\
0, & \text { otherwise }
\end{array},\right.
$$

we have a straightforward lemma.

Lemma 3. Let $(p, r)$ be a binary fingerprinting strategy. Then $P_{\mathrm{wce}}^{(p, r)}=1$ iff $I_{x}^{(p)} \subseteq I_{y}^{(p)}$ for some $x \neq y$.

We now restate Lemma 3 in the language of extremal set theory $[13,14,15]$. Roger's decision matrix may be specified by the set $\mathcal{I}^{(p)} \equiv\left\{I_{x}^{(p)} \mid x=1, \ldots, n\right\}$ which is a subset of the power set $2^{[m]}$, where $[m] \equiv\{1, \ldots, m\}$ is called the $m$-set. A $k$-subset of a set $X$ is simply a subset of $X$ with cardinality $k$, and the set of all such subsets will be denoted by the symbol $\left(\begin{array}{l}X \\ k\end{array}\right)$. Define an antichain $\mathcal{A}$ of the poset $(\mathcal{S}, \subseteq)$ to be a subset of $\mathcal{S}$ with the property that no two members are comparable via the relation $\subseteq$, i.e. $X \nsubseteq Y$ for all distinct $X, Y \in \mathcal{A}$. The length of the antichain is its cardinality, $|\mathcal{A}|$. The following is now equivalent to Lemma 3.

Lemma $3^{\prime}$ 1. Let $(p, r)$ be a binary fingerprinting strategy. Then $P_{\mathrm{wce}}^{(p, r)}<1$ iff $\mathcal{I}^{(p)}$ is an antichain of length $n$ in $\left(2^{[m]}, \subseteq\right)$.

An example of an antichain is $\{\{1,2\},\{1,3\},\{1,4\},\{2,3\},\{2,4\},\{3,4\}\}$, which is also the largest antichain in $2^{[4]}$, and most easily represented through its binary incidence matrix:

\begin{tabular}{llll}
1 & 2 & 3 & 4 \\
\hline 1 & 1 & 0 & 0 \\
1 & 0 & 1 & 0 \\
1 & 0 & 0 & 1 \\
0 & 1 & 1 & 0 \\
0 & 1 & 0 & 1 \\
0 & 0 & 1 & 1
\end{tabular}.


In our case this incidence matrix is in fact $r(a, x)$, where $a$ indexes the columns and $x$ the rows. The above antichain has length 6 . In general, the length of the largest antichain in $2^{[\mathrm{m}]}$ is $\left(\begin{array}{c}m \\ \lfloor m / 2\rfloor\end{array}\right)$. This result is due to Sperner [16] and gives us our first important theorem:

Theorem 4. $\mathcal{P}_{\text {wce }}^{1}(n, m, n)<1$ iff $n \leq\left(\begin{array}{c}m \\ \lfloor m / 2\rfloor\end{array}\right)$.

When $m<n \leq\left(\begin{array}{c}m \\ \lfloor m / 2\rfloor\end{array}\right)$ an optimal strategy can be found by first collecting all antichains of length $n$, and then for each such candidate for $\mathcal{I}^{(p)}$, minimize $P_{\text {wce }}^{(p, r)}$ over Alice's transition probabilities $p(a \mid x)$ to locate the optimal choice. Consider the case when $n=\left(\begin{array}{c}m \\ \lfloor m / 2\rfloor\end{array}\right)$ for example. If $m$ is even, the set of all $m / 2$-subsets of the $m$-set, $\left(\begin{array}{c}{[m]} \\ m / 2\end{array}\right)$, is the only antichain; if $m$ is odd, the sets $\left(\begin{array}{c}{[m]} \\ (m-1) / 2\end{array}\right)$ and $\left(_{(m+1) / 2}^{[m]}\right)$ are the only antichains $[13,17]$. In both cases an optimal strategy follows from choosing $\mathcal{I}^{(p)}=\left\{I_{x}^{(p)} \mid x=1, \ldots, n\right\}=\left(\begin{array}{c}{[\mathrm{m}]} \\ \lfloor\mathrm{m} / 2\rfloor\end{array}\right)$ and then $p(a \mid x)=1 /\lfloor m / 2\rfloor$ when $a \in I_{x}^{(p)}$ (and zero otherwise). For example, when $m=4$ and $n=\left(\begin{array}{l}4 \\ 2\end{array}\right)=6$ the above antichain [Eq. (18)] is the only choice, in which case setting $p(a \mid x)=$ $r(a, x) / 2$ is optimal, resulting in $P_{\text {wce }}^{(p, r)}=1 / 2$. In general, the resulting worst-case error probability is stated in the following proposition.

Proposition 5. $\mathcal{P}_{\text {wce }}^{1}(n, m, n)=1-1 /\lfloor m / 2\rfloor$ if $n=\left(\begin{array}{c}m \\ \lfloor m / 2\rfloor\end{array}\right)$.

Searching for antichains is computationally expensive except for small $m$ and $n$. We instead turn our attention to deriving upper and lower bounds for $\mathcal{P}_{\text {wce }}^{1}(n, m, n)$. Lower bounds follow from generalizing the concept of an antichain to a $k$-cover free family of sets [18, 19]. A family of sets is simply a set of sets. We call the family $\mathcal{F}, k$-cover free when $X \nsubseteq Y_{1} \cup \cdots \cup Y_{k}$ for all $X, Y_{1}, \ldots, Y_{k} \in \mathcal{F}$, where $X \neq Y_{i}$ for all $i$. Note that a 1 -cover free family is an antichain. The following incidence matrix specifies an example of a 2-cover free family.

\begin{tabular}{lllllllll}
1 & 2 & 3 & 4 & 5 & 6 & 7 & 8 & 9 \\
\hline 1 & 1 & 1 & 0 & 0 & 0 & 0 & 0 & 0 \\
0 & 0 & 0 & 1 & 1 & 1 & 0 & 0 & 0 \\
0 & 0 & 0 & 0 & 0 & 0 & 1 & 1 & 1 \\
1 & 0 & 0 & 1 & 0 & 0 & 1 & 0 & 0 \\
0 & 1 & 0 & 0 & 1 & 0 & 0 & 1 & 0 \\
0 & 0 & 1 & 0 & 0 & 1 & 0 & 0 & 1 \\
1 & 0 & 0 & 0 & 1 & 0 & 0 & 0 & 1 \\
0 & 1 & 0 & 0 & 0 & 1 & 1 & 0 & 0 \\
0 & 0 & 1 & 1 & 0 & 0 & 0 & 1 & 0 \\
1 & 0 & 0 & 0 & 0 & 1 & 0 & 1 & 0 \\
0 & 1 & 0 & 1 & 0 & 0 & 0 & 0 & 1 \\
0 & 0 & 1 & 0 & 1 & 0 & 1 & 0 & 0
\end{tabular}

Suppose that Alice uses a strategy where $I_{x}^{(p)} \subseteq I_{y}^{(p)} \cup I_{z}^{(p)}$ for some $x \neq y, z$. Then, for one choice between the message pairs $(x, y)$ and $(x, z)$, Sapna will be able to coerce an error probability of $1 / 2$ or greater. This follows from the inequality

$$
P_{1}^{(p, r)}(x, y)+P_{1}^{(p, r)}(x, z)=\sum_{a=1}^{m} p(a \mid x)[r(a, y)+r(a, z)] \geq \sum_{a=1}^{m} p(a \mid x) r(a, x)=1,
$$

since the assumption $I_{x}^{(p)} \subseteq I_{y}^{(p)} \cup I_{z}^{(p)}$ implies that either $r(a, y)=1$ or $r(a, z)=1$ when $r(a, x)=1$. Thus the worst-case error probability may be less than $1 / 2$ only when $\mathcal{I}^{(p)}$ is a 


\begin{tabular}{|c|ccccccccccccccc|}
\hline$q \backslash m$ & 2 & 3 & 4 & 5 & 6 & 7 & 8 & 9 & 10 & 11 & 12 & 13 & 14 & 15 & 16 \\
\hline 1 & 2 & 3 & 6 & 10 & 20 & 35 & 70 & 126 & 252 & 462 & 924 & 1716 & 3432 & 6435 & 12870 \\
$4 / 3$ & 2 & 3 & 6 & 10 & 20 & 35 & $\geq 56$ & $\geq 84$ & $\geq 120$ & $\geq 165$ & $\geq 220$ & $\geq 286$ & $\geq 364$ & $\geq 455$ & $\geq 560$ \\
$3 / 2$ & 2 & 3 & 6 & 10 & 15 & 21 & $\geq 28$ & $\geq 36$ & $\geq 45$ & $\geq 55$ & $\geq 66$ & $\geq 78$ & $\geq 91$ & $\geq 105$ & $\geq 120$ \\
2 & 2 & 3 & 4 & 5 & 6 & 7 & 8 & 12 & 13 & $\geq 17$ & $\geq 20$ & $\geq 26$ & $\geq 28$ & $\geq 35$ & $\geq 37$ \\
3 & 2 & 3 & 4 & 5 & 6 & 7 & 8 & 9 & 10 & 11 & 12 & 13 & 14 & 15 & $\geq 20$ \\
4 & 2 & 3 & 4 & 5 & 6 & 7 & 8 & 9 & 10 & 11 & 12 & 13 & 14 & 15 & $\geq 16$ \\
\hline
\end{tabular}

Table 1. Known values of $T(m, q)$, the largest $q$-cover free family in $2^{[m]}$, or a lower bound when unknown.

2-cover free family. This observation, in its generalized form, is the content of the following lemma.

Lemma 6. Let $(p, r)$ be a binary fingerprinting strategy. Then $P_{\mathrm{wce}}^{(p, r)}<1 / k$ only if $\mathcal{I}^{(p)}$ is a $k$-cover free family of size $n$ in $2^{[m]}$.

Unlike in the case of antichains, the size of the largest $k$-cover free family in the power set $2^{[m]}$ is a difficult problem, and consequently, unknown except in a few special cases. Nonetheless, let us denote this number by $T(m, k)$. The following theorem then applies.

Theorem 7. $\mathcal{P}_{\text {wce }}^{1}(n, m, n) \geq 1 / k$ if $n>T(m, k)$.

Corollary 8. $\mathcal{P}_{\text {wce }}^{1}(n, m, n) \geq 1 / k$ if $\left(\begin{array}{l}n \\ k\end{array}\right)>\left(\begin{array}{c}m \\ \lfloor m / 2\rfloor\end{array}\right)$.

The corollary follows from the fact that a family $\mathcal{F}$ is $k$-cover free only if the set $\left\{Y_{1} \cup\right.$ $\cdots \cup Y_{k} \mid$ distinct $\left.Y_{1}, \ldots, Y_{k} \in \mathcal{F}\right\}$ forms an antichain. The obtained bound, however, may be tightened by calculating the exact value of $T(m, k)$. This problem is also known under the name of superimposed codes [20]. In fact, each $k$-cover free family of sets $\mathcal{F}$ is equivalent to a superimposed code, in which the binary incidence vectors corresponding to members of $\mathcal{F}$ form the codewords. The exact value of $T(m, k)$ is known when $k=1$, in which case Sperner's result for the largest antichain applies, and for the following special cases. Tables from [21] show that $T(m, 2)=m$ when $m \leq 8$, and $T(m, k)=m$ when $m \leq 15$ and $k \geq 3$. Also, our own computational searches have found that $T(9,2)=12$ and $T(10,2)=13$. These results are tabulated in Table 1 (integral $q$ ), and give many of the lower bounds in Table 2 for $\mathcal{P}_{\text {wce }}^{1}(n, m, n)$.

We can generalize Theorem 7 by introducing the concept of a multiset. A multiset is like a set, except that repeated elements are now allowed, e.g. $X=\{1,1,2,3\}$. Multiset operations then differ from set operations in a straightforward way: $X \cup\{2\}=\{1,1,2,2,3\}$, $X \cap\{1,1,2,2\}=\{1,1,2\}$, and $\{1,1\} \subseteq X$ but $\{1,1,1\} \nsubseteq \subseteq X$. Let $\cup^{j} X$ denote the multiset union of $j$ copies of $X$. A family of sets $\mathcal{F}$ will be called $k / j$-cover free when $\cup^{j} X \nsubseteq \subseteq Y_{1} \cup \cdots \cup Y_{k}$, under multiset operations, for all $X, Y_{1}, \ldots, Y_{k} \in \mathcal{F}$, where $X \neq Y_{i}$ for all $i$. Note that a family of sets is $q$-cover free for all rationals $q \leq p$ whenever it is $p$-cover free. An example of a $3 / 2$ cover free family is the set $\left(\begin{array}{c}{[6]} \\ 2\end{array}\right)$.

Lemma 9. Let $(p, r)$ be a binary fingerprinting strategy. Then $P_{\mathrm{wce}}^{(p, r)}<1 / q$ only if $\mathcal{I}^{(p)}$ is a q-cover free family of size $n$ in $2^{[m]}$.

This lemma follows from a straightforward generalization of Eq. (20): Suppose now that 


\begin{tabular}{|c|cccccccccccc|}
\hline$n \backslash m$ & 2 & 3 & 4 & 5 & 6 & 7 & 8 & 9 & 10 & 11 & 12 & 13 \\
\hline 2 & 0 & 0 & 0 & 0 & 0 & 0 & 0 & 0 & 0 & 0 & 0 & 0 \\
3 & 1 & 0 & 0 & 0 & 0 & 0 & 0 & 0 & 0 & 0 & 0 & 0 \\
4 & 1 & 1 & 0 & 0 & 0 & 0 & 0 & 0 & 0 & 0 & 0 & 0 \\
5 & 1 & 1 & $1 / 2$ & 0 & 0 & 0 & 0 & 0 & 0 & 0 & 0 & 0 \\
6 & 1 & 1 & $1 / 2$ & $1 / 2$ & 0 & 0 & 0 & 0 & 0 & 0 & 0 & 0 \\
7 & 1 & 1 & 1 & $1 / 2$ & $1 / 2$ & 0 & 0 & 0 & 0 & 0 & 0 & 0 \\
8 & 1 & 1 & 1 & $1 / 2$ & $1 / 2$ & $1 / 2$ & 0 & 0 & 0 & 0 & 0 & 0 \\
9 & 1 & 1 & 1 & $1 / 2$ & $1 / 2$ & $1 / 2$ & $1 / 2$ & 0 & 0 & 0 & 0 & 0 \\
10 & 1 & 1 & 1 & $1 / 2$ & $1 / 2$ & $1 / 2$ & $1 / 2$ & $1 / 3$ & 0 & 0 & 0 & 0 \\
11 & 1 & 1 & 1 & 1 & $1 / 2$ & $1 / 2$ & $1 / 2$ & $1 / 3$ & $1 / 3$ & 0 & 0 & 0 \\
12 & 1 & 1 & 1 & 1 & $1 / 2$ & $1 / 2$ & $1 / 2$ & $1 / 3$ & $1 / 3$ & $1 / 3$ & 0 & 0 \\
13 & 1 & 1 & 1 & 1 & $1 / 2$ & $1 / 2$ & $1 / 2$ & $1 / 2$ & $1 / 3$ & $1 / 3$ & $1 / 3$ & 0 \\
14 & 1 & 1 & 1 & 1 & $1 / 2$ & $1 / 2$ & $1 / 2$ & $1 / 2$ & $1 / 2$ & $1 / 3$ & $1 / 3$ & $1 / 3$ \\
15 & 1 & 1 & 1 & 1 & $1 / 2$ & $1 / 2$ & $1 / 2$ & $1 / 2$ & $1 / 2$ & $1 / 3$ & $1 / 3$ & $1 / 3$ \\
16 & 1 & 1 & 1 & 1 & $2 / 3$ & $1 / 2$ & $1 / 2$ & $1 / 2$ & $1 / 2$ & $1 / 3$ & $1 / 3$ & $1 / 3$ \\
17 & 1 & 1 & 1 & 1 & $2 / 3$ & $1 / 2$ & $1 / 2$ & $1 / 2$ & $1 / 2$ & $1 / 3$ & $1 / 3$ & $1 / 3$ \\
18 & 1 & 1 & 1 & 1 & $2 / 3$ & $1 / 2$ & $1 / 2$ & $1 / 2$ & $1 / 2$ & $1 / 3-1 / 2$ & $1 / 3$ & $1 / 3$ \\
19 & 1 & 1 & 1 & 1 & $2 / 3$ & $1 / 2$ & $1 / 2$ & $1 / 2$ & $1 / 2$ & $1 / 3-1 / 2$ & $1 / 3$ & $1 / 3$ \\
20 & 1 & 1 & 1 & 1 & $2 / 3$ & $1 / 2$ & $1 / 2$ & $1 / 2$ & $1 / 2$ & $1 / 3-1 / 2$ & $1 / 3$ & $1 / 3$ \\
21 & 1 & 1 & 1 & 1 & 1 & $1 / 2$ & $1 / 2$ & $1 / 2$ & $1 / 2$ & $1 / 3-1 / 2$ & $1 / 3-1 / 2$ & $1 / 3$ \\
22 & 1 & 1 & 1 & 1 & 1 & $2 / 3$ & $1 / 2$ & $1 / 2$ & $1 / 2$ & $1 / 3-1 / 2$ & $1 / 3-1 / 2$ & $1 / 3$ \\
\hline
\end{tabular}

Table 2. $\mathcal{P}_{\text {wce }}^{1}(n, m, n)$, the minimum achievable worst-case error probability for a classical fingerprinting strategy under the one-way communication model, or the range of possible values when unknown.

$\cup^{j} I_{x}^{(p)} \subseteq I_{y_{1}}^{(p)} \cup \cdots \cup I_{y_{k}}^{(p)}$, under multiset operations, for some $x \neq y_{1}, \ldots, y_{k}$. Then

$$
\begin{aligned}
P_{1}^{(p, r)}\left(x, y_{1}\right)+\cdots+P_{1}^{(p, r)}\left(x, y_{k}\right) & =\sum_{a=1}^{m} p(a \mid x)\left[r\left(a, y_{1}\right)+\cdots+r\left(a, y_{k}\right)\right] \\
& \geq j \sum_{a=1}^{m} p(a \mid x) r(a, x) \\
& =j
\end{aligned}
$$

since the condition $\cup^{j} I_{x}^{(p)} \subseteq I_{y_{1}}^{(p)} \cup \cdots \cup I_{y_{k}}^{(p)}$ means that $r\left(a, y_{l}\right)=1$ whenever $r(a, x)=1$ for $j$ different choices of $1 \leq l \leq k$. Equation (23) implies that $P_{1}^{(p, r)}\left(x, y_{l}\right) \geq j / k$ for at least one $l$, and thus the worst-case error probability can be less than $j / k$ only when $\mathcal{I}^{(p)}$ is a $k / j$-cover free family.

For rational $q$, if we let $T(m, q)$ denote the size of the largest $q$-cover free family in the power set $2^{[m]}$, then Theorem 7 generalizes to the following.

Theorem 10. $\mathcal{P}_{\text {wce }}^{1}(n, m, n) \geq 1 / q$ if $n>T(m, q)$.

Our own computational searches have found that $T(m, 3 / 2)=\left(\begin{array}{c}m \\ 2\end{array}\right)$ for $m \leq 7$, which is also tabulated in Table 1, and gives the corresponding lower bounds on $\mathcal{P}_{\text {wce }}^{1}(n, m, n)$ in Table 2. This formula, however, is unlikely to be true in general.

We obtain an upper bound on $\mathcal{P}_{\text {wce }}^{1}(n, m, n)$ whenever a particular example strategy is found. Consider those derived from constant weight codes $[22,23]$. Recall that the weight of a binary vector $\mathbf{u}=\left\{u_{i}\right\}_{i=1}^{n}$ is $\operatorname{wt}(\mathbf{u})=\sum_{i} u_{i}$, and the Hamming distance between two vectors $\mathbf{u}$ and $\mathbf{v}$ is $\mathrm{d}(\mathbf{u}, \mathbf{v})=\sum_{i}\left|u_{i}-v_{i}\right|$. The code-theoretic function $A(n, d, w)$ is defined as the maximum possible number of binary vectors of length $n$, Hamming distance at least $d$ apart, and constant weight $w$. If we let $N(m, k, j)$ denote the maximum number of $k$-subsets 
of the $m$-set with pairwise intersections containing $j$ elements or less, then $N(m, k, j)=$ $A(m, 2(k-j), k)$. We can see this by considering the codewords as incidence vectors for subsets of the $m$-set, thus defining the isomorphism $f:\{0,1\}^{m} \rightarrow 2^{[m]}$ by $f(\mathbf{u})=\left\{i \mid u_{i}=1\right\}$, and noting that $\mathrm{d}(\mathbf{u}, \mathbf{v})=2(k-|f(\mathbf{u}) \cap f(\mathbf{v})|)$ when $\operatorname{wt}(\mathbf{u})=\operatorname{wt}(\mathbf{v})=k$. Now given a family $\mathcal{F}$, of $n k$-subsets of the $m$-set with pairwise intersections containing $j$ elements or less, we may construct a strategy with $P_{\text {wce }}^{(p, r)} \leq j / k$ by uniquely assigning each index matrix $I_{x}^{(p)}(x=1, . ., n)$ with a member of $\mathcal{F}$ and setting $p(a \mid x)=1 / k$ when $a \in I_{x}^{(p)}$ (and zero otherwise). Thus $\mathcal{P}_{\text {wce }}^{1}(n, m, n) \leq j / k$ whenever $n \leq N(m, k, j)=A(m, 2(k-j), k)$, which is restated in the following theorem.

Theorem 11. $\mathcal{P}_{\text {wce }}^{1}(n, m, n) \leq j / k$ if $n \leq N(m, k, j)=A(m, 2(k-j), k)$.

In some cases $A(n, d, w)$ is known exactly [22]. Examples are $N(m, k, k-1)=A(m, 2, k)=$ $\left(\begin{array}{c}m \\ k\end{array}\right)$, which is trivial,

$$
N(m, 3,1)=A(m, 4,3)=\left\{\begin{array}{lll}
\left\lfloor\frac{m}{3}\left\lfloor\frac{m-1}{2}\right\rfloor\right\rfloor, & \text { if } m \not \equiv 5 & \bmod 6 \\
\left\lfloor\frac{m}{3}\left\lfloor\frac{m-1}{2}\right\rfloor\right\rfloor-1, & \text { if } m \equiv 5 \bmod 6
\end{array}\right.
$$

and

$$
N\left(q^{2}, q, 1\right)=A\left(q^{2}, 2 q-2, q\right)=q(q+1)
$$

if $q$ is a prime power. These results give the upper bounds on $\mathcal{P}_{\text {wce }}^{1}(n, m, n)$ in Table 2. Thus far, we do not know of a strategy with smaller worst-case error probability than that attained by a constant-weight-code strategy.

Finally, we remark that the inequalities $\mathcal{P}_{\text {wce }}^{1}(n+1, m, n+1) \geq \mathcal{P}_{\text {wce }}^{1}(n, m, n) \geq \mathcal{P}_{\text {wce }}^{1}(n+$ $1, m+1, n+1)$ trivially hold, since increasing the number of messages will not allow lower error probabilities, and a strategy with $n+1$ messages and $m+1$ fingerprints can be constructed from one with $n$ messages and $m$ fingerprints, by simply allowing Alice to pass on the additional message to Roger as the additional fingerprint.

\subsection{Generalizations and the SMP model: $m_{A}=m_{B}$}

We now apply some of the ideas of the previous section to the general case where Bob's fingerprint set is also restricted in size. Defining index sets for Alice's and Bob's fingerprinting strategies

$$
I_{x}^{(p)} \equiv\{a \mid p(a \mid x)>0\}, \quad I_{y}^{(q)} \equiv\{b \mid q(b \mid y)>0\},
$$

a binary strategy is one with

$$
r(a, b)=\left\{\begin{array}{ll}
1, & \text { if }(a, b) \in \cup_{z} I_{z}^{(p)} \times I_{z}^{(q)} \\
0, & \text { otherwise }
\end{array} .\right.
$$

After inspection of Eq. (3) it is straightforward to see that Lemma 3 of the previous section takes the following generalized form.

Lemma 12. Let $(p, q, r)$ be a binary fingerprinting strategy. Then $P_{\mathrm{wce}}^{(p, q, r)}=1$ iff $I_{x}^{(p)} \times I_{y}^{(q)} \subseteq$ $\cup_{z} I_{z}^{(p)} \times I_{z}^{(q)}$ for some $x \neq y$.

A necessary condition for $P_{\text {wce }}^{(p, q, r)}<1$ is to have both families of sets $\mathcal{I}^{(p)}=\left\{I_{x}^{(p)}\right\}$ and $\mathcal{I}^{(q)}=\left\{I_{y}^{(q)}\right\}$ forming antichains. Note however, that a different labelling of members in one 
of the families (through subscript $x$ or $y$ ) will, in general, correspond to a different strategy; consequently, the enumeration of elements in $\mathcal{I}^{(p)}$ and $\mathcal{I}^{(q)}$ is now important.

Lemma 12 may be used to derive conditions upon which the worst-case error probability is necessarily 1 , thus generalizing Theorem 4 of the previous section. We may also generalize Theorem 4, however, in the following straightforward manner. Setting $m=\min \left\{m_{A}, m_{B}\right\}$, we first note that Theorem 4 implies $\mathcal{P}_{\text {wce }}^{1}\left(n, m_{A}, m_{B}\right)=1$ whenever $n>\left(\begin{array}{c}m \\ \lfloor m / 2\rfloor\end{array}\right)$, since the minimum possible worst-case error probability cannot decrease when the size of Bob's message pool, $m_{B}$, is decreased. We now claim that in fact the converse is also true. That is, whenever $\mathcal{P}_{\text {wce }}^{1}\left(n, m_{A}, m_{B}\right)=1$ we must have $n>\left(\begin{array}{c}m \\ \lfloor m / 2\rfloor\end{array}\right)$, or equivalently, $\mathcal{P}_{\text {wce }}^{1}(n, m, m)<1$ when $n=\left(\begin{array}{c}m \\ \lfloor m / 2\rfloor\end{array}\right)$. Explicit examples of such strategies will prove our claim. Consider the binary strategies with $\mathcal{I}^{(p)}=\left(\begin{array}{c}{[m]} \\ {[m / 2\rfloor}\end{array}\right)$ (any labelling of elements in this family will do) and then $I_{y}^{(q)} \equiv I_{y}^{(p)^{\prime}}$, the complement of $I_{y}^{(p)}$ in $[m]$. Roger's decisions are dictated by Eq. (27), and hence, $r(a, b)=1-\delta(a, b)$. Finally by choosing, for example, $p(a \mid x)=1 /\lfloor m / 2\rfloor$ when $a \in I_{x}^{(p)}$ (otherwise zero), and $q(b \mid y)=1 /\lceil m / 2\rceil$ when $b \in I_{y}^{(q)}=I_{y}^{(p)^{\prime}}$, we find that $P_{\mathrm{wce}}^{(p, q, r)}=1-1 /(\lfloor m / 2\rfloor\lceil m / 2\rceil)<1$ and the following theorem is proven.

Theorem 13. $\mathcal{P}_{\text {wce }}^{1}\left(n, m_{A}, m_{B}\right)<1$ iff $n \leq\left(\begin{array}{c}m \\ \lfloor m / 2\rfloor\end{array}\right)$ where $m=\min \left\{m_{A}, m_{B}\right\}$.

The generalization of other results from the previous section was found to be quite difficult. We can say some more, however, about the special case of Alice and Bob sharing the same set of fingerprints, i.e. when $m_{A}=m_{B}=m$. In particular, the strategy given above can be shown to be optimal:

Proposition 14. $\mathcal{P}_{\text {wce }}^{1}(n, m, m)=1-1 /(\lfloor m / 2\rfloor\lceil m / 2\rceil)$ if $n=\left(\begin{array}{c}m \\ \lfloor m / 2\rfloor\end{array}\right)$.

To prove this result, consider the case when $m$ is even. Since $n=\left(\begin{array}{c}m \\ m / 2\end{array}\right)$, the only choice for $\mathcal{I}^{(p)}$ and $\mathcal{I}^{(q)}$ is the antichain $\left(\begin{array}{c}{[m]} \\ m / 2\end{array}\right)$. We must now decide how to optimally label the index sets $I_{x}^{(p)} \in \mathcal{I}^{(p)}$ and $I_{y}^{(q)} \in \mathcal{I}^{(q)}$. There is in fact only one choice, modulo permutations of message and fingerprint labels, for which $I_{x}^{(p)} \times I_{y}^{(q)} \not \subset \cup_{z} I_{z}^{(p)} \times I_{z}^{(q)}$ for every $x \neq y$, and by Lemma 12, allows a worst-case error probability less than 1 . We can show this as follows. Suppose that $r(m, m)=0$ as in the strategy given immediately above. Notice that fingerprint $m$ belongs to exactly $n / 2$ members of the antichain $\left(\begin{array}{c}{[m]} \\ m / 2\end{array}\right)$, and thus, exactly $n / 2$ members of $\mathcal{I}^{(p)}$, which without loss of generality are chosen to be $I_{1}^{(p)}, \ldots, I_{n / 2}^{(p)}$. Consequently, by Eq. (27), if $r(m, m)=0$ then fingerprint $m$ can not belong to any of $I_{1}^{(q)}, \ldots, I_{n / 2}^{(q)}$. The only

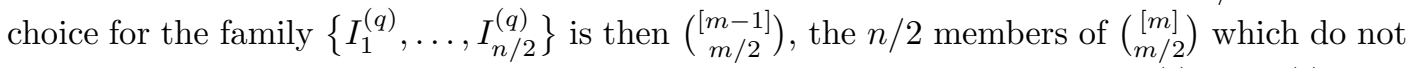
contain $m$. But we now have each of the fingerprints $1, \ldots, m-1$ in one of $I_{1}^{(q)}, \ldots, I_{n / 2}^{(q)}$, and fingerprint $m$ in all of $I_{1}^{(p)}, \ldots, I_{n / 2}^{(p)}$. Thus, by Eq. $(27), r(m, b)=1$ for all $1 \leq b \leq m-1$. By symmetry, the same can be said about the last column of the matrix $r(a, b)$, i.e. $r(a, m)=1$ for all $1 \leq a \leq m-1$. Furthermore, there was nothing special about our choice to set $r(m, m)=0$. We can summarize this result in the following way. For any labelling of the index sets, every row and every column of the $m \times m$ matrix $r(a, b)$ has at least $m-1$ entries equal to 1 . The condition for the worst-case error probability to be less than 1 can be satisfied only for those matrices with a 0 in every row and every column. Thus $r(a, b)$ must be a row/column permutation of the $m \times m$ matrix with a diagonal of 0 's, and off-diagonal elements equal to 1 . Such strategies correspond to a fingerprint relabelling of the strategy 


\begin{tabular}{|c|cccccccc|}
\hline$n \backslash m$ & 2 & 3 & 4 & 5 & 6 & 7 & 8 & 9 \\
\hline 2 & 0 & 0 & 0 & 0 & 0 & 0 & 0 & 0 \\
3 & 1 & 0 & 0 & 0 & 0 & 0 & 0 & 0 \\
4 & 1 & 1 & 0 & 0 & 0 & 0 & 0 & 0 \\
5 & 1 & 1 & $3 / 4$ & 0 & 0 & 0 & 0 & 0 \\
6 & 1 & 1 & $3 / 4$ & $1 / 2-3 / 4$ & 0 & 0 & 0 & 0 \\
7 & 1 & 1 & 1 & $1 / 2-3 / 4$ & $1 / 2-3 / 4$ & 0 & 0 & 0 \\
8 & 1 & 1 & 1 & $1 / 2-3 / 4$ & $1 / 2-3 / 4$ & $1 / 2-3 / 4$ & 0 & 0 \\
9 & 1 & 1 & 1 & $5 / 6$ & $1 / 2-3 / 4$ & $1 / 2-3 / 4$ & $1 / 2-3 / 4$ & 0 \\
10 & 1 & 1 & 1 & $5 / 6$ & $1 / 2-3 / 4$ & $1 / 2-3 / 4$ & $1 / 2-3 / 4$ & $1 / 3-1 / 2$ \\
11 & 1 & 1 & 1 & 1 & $1 / 2-3 / 4$ & $1 / 2-3 / 4$ & $1 / 2-3 / 4$ & $1 / 3-3 / 4$ \\
12 & 1 & 1 & 1 & 1 & $1 / 2-3 / 4$ & $1 / 2-3 / 4$ & $1 / 2-3 / 4$ & $1 / 3-3 / 4$ \\
13 & 1 & 1 & 1 & 1 & $1 / 2-5 / 6$ & $1 / 2-3 / 4$ & $1 / 2-3 / 4$ & $1 / 2-3 / 4$ \\
14 & 1 & 1 & 1 & 1 & $1 / 2-8 / 9$ & $1 / 2-3 / 4$ & $1 / 2-3 / 4$ & $1 / 2-3 / 4$ \\
15 & 1 & 1 & 1 & 1 & $1 / 2-8 / 9$ & $1 / 2-3 / 4$ & $1 / 2-3 / 4$ & $1 / 2-3 / 4$ \\
16 & 1 & 1 & 1 & 1 & $2 / 3-8 / 9$ & $1 / 2-3 / 4$ & $1 / 2-3 / 4$ & $1 / 2-3 / 4$ \\
17 & 1 & 1 & 1 & 1 & $2 / 3-8 / 9$ & $1 / 2-3 / 4$ & $1 / 2-3 / 4$ & $1 / 2-3 / 4$ \\
18 & 1 & 1 & 1 & 1 & $2 / 3-8 / 9$ & $1 / 2-3 / 4$ & $1 / 2-3 / 4$ & $1 / 2-3 / 4$ \\
19 & 1 & 1 & 1 & 1 & $2 / 3-8 / 9$ & $1 / 2-3 / 4$ & $1 / 2-3 / 4$ & $1 / 2-3 / 4$ \\
20 & 1 & 1 & 1 & 1 & $8 / 9$ & $1 / 2-3 / 4$ & $1 / 2-3 / 4$ & $1 / 2-3 / 4$ \\
21 & 1 & 1 & 1 & 1 & 1 & $1 / 2-3 / 4$ & $1 / 2-3 / 4$ & $1 / 2-3 / 4$ \\
22 & 1 & 1 & 1 & 1 & 1 & $2 / 3-8 / 9$ & $1 / 2-3 / 4$ & $1 / 2-3 / 4$ \\
\hline
\end{tabular}

Table 3. $\mathcal{P}_{\text {wce }}^{1}(n, m, m)$, the minimum achievable worst-case error probability for a classical fingerprinting strategy under the SMP model, or the range of possible values when unknown.

given above, and are optimal when the nonzero transmission probabilities of $p(b \mid x)$ and $q(b \mid y)$ are symmetrically chosen to be $2 / m$. The case when $m$ is odd is similar.

In principle, we can also use Lemma 12 in this way to locate an optimal strategy for other $n$. One must, however, test all possible choices for $\mathcal{I}^{(p)}$ and $\mathcal{I}^{(q)}$, i.e., all antichains of length $n$, which grow exponentially in number as $n$ decreases. Consequently, this laborious task was undertaken only for two special cases, with results: $\mathcal{P}_{\text {wce }}^{1}(5,4,4)=3 / 4$ and $\mathcal{P}_{\text {wce }}^{1}(9,5,5)=5 / 6$.

In Table 3 we report the known values of $\mathcal{P}_{\text {wce }}^{1}(n, m, m)$, or the range of possible values when unknown. Apart from the above special cases where the exact value of $\mathcal{P}_{\text {wce }}^{1}(n, m, m)$ was derived, all lower bounds are the same as those given in the previous section, being derived from the inequality $\mathcal{P}_{\text {wce }}^{1}(n, m, m) \geq \mathcal{P}_{\text {wce }}^{1}(n, m, n)$. Such bounds are likely to be weak in general.

The upper bounds on $\mathcal{P}_{\text {wce }}^{1}(n, m, m)$ in Table 3 come from explicit strategies. First note that we will always have $\mathcal{P}_{\text {wce }}^{1}(2 n, m+n, m+n) \leq \mathcal{P}_{\text {wce }}^{1}(n, m, n)$. This follows from a straightforward conversion of an $(n, m, n)$-fingerprinting strategy of the previous section, denoted by $\left(p^{\prime}, r^{\prime}\right)$ say, into a strategy suitable for employment in the current scenario: Previously, Alice performed the fingerprinting strategy $p^{\prime}$, while Bob simply acted as a relay, and passed his message on to Roger. To covert this strategy into one suitable for the current scenario, we ask Alice to employ $p^{\prime}$ on the first half of Sapna's messages and relay the second half, while Bob is tasked with the opposite, fingerprinting on the second half and relaying on the first. Explicitly,

$$
\begin{aligned}
& p(a \mid x)= \begin{cases}p^{\prime}(a \mid x), & \text { if } 1 \leq a \leq m \text { and } 1 \leq x \leq n \\
\delta(a-m, x-n), & \text { otherwise }\end{cases} \\
& q(b \mid y)= \begin{cases}p^{\prime}(b \mid y-n), & \text { if } 1 \leq b \leq m \text { and } n+1 \leq y \leq 2 n \\
\delta(b-m, y), & \text { otherwise }\end{cases}
\end{aligned}
$$


Roger may now always discern which half of the message set $(\{1, \ldots, n\}$ or $\{n+1, \ldots, 2 n\})$ that $x$ and $y$ belong to, by simply noting which subdivision $a$ and $b$ belong to $(\{1, \ldots, m\}$ or $\{m+1, \ldots, m+n\})$, and immediately calls 0 when different, or performs $r^{\prime}$ appropriately when the same. The resulting worst-case error probability is then equal to that for the $(n, m, n)$ strategy $\left(p^{\prime}, r^{\prime}\right)$. In Table 3 the upper bound $\mathcal{P}_{\text {wce }}^{1}(10,9,9) \leq \mathcal{P}_{\text {wce }}^{1}(5,4,5)=1 / 2$ comes from this method.

To obtain further upper bounds we can consider a variation of the constant-weight-code strategies given in Sec. 2.2: If $\mathcal{I}^{(p)}=\left\{I_{x}^{(p)}\right\}$ and $\mathcal{I}^{(q)}=\left\{I_{y}^{(q)}\right\}$ are ordered families of, respectively, $k_{1}$ - and $k_{2}$-subsets of the $m$-set, both of size $n$, with the property that

$$
\left|\left(I_{x}^{(p)} \times I_{y}^{(q)}\right) \cap\left(\cup_{z} I_{z}^{(p)} \times I_{z}^{(q)}\right)\right| \leq j
$$

for all $x \neq y$, then $\mathcal{P}_{\text {wce }}^{1}(n, m, m) \leq j /\left(k_{1} k_{2}\right)$. The strategies which achieve this worst-case error probability are defined by simply setting $p(a \mid x)=1 / k_{1}$ when $a \in I_{x}^{(p)}$ (zero otherwise), and $q(b \mid y)=1 / k_{2}$ when $b \in I_{y}^{(q)}$. Let us denote by $N_{2}\left(m, k_{1}, k_{2}, j\right)$ the largest $n$ such that a pair of such families exist. The following theorem is then a variation of Theorem 11.

Theorem 15. $\mathcal{P}_{\text {wce }}^{1}(n, m, m) \leq j /\left(k_{1} k_{2}\right)$ if $n \leq N_{2}\left(m, k_{1}, k_{2}, j\right)$.

Our own computational searches have found that $N_{2}(5,2,2,3)=8$. A strategy with the corresponding worst-case error probability of $3 / 4$ is defined through the pair of ordered families with incidence matrices

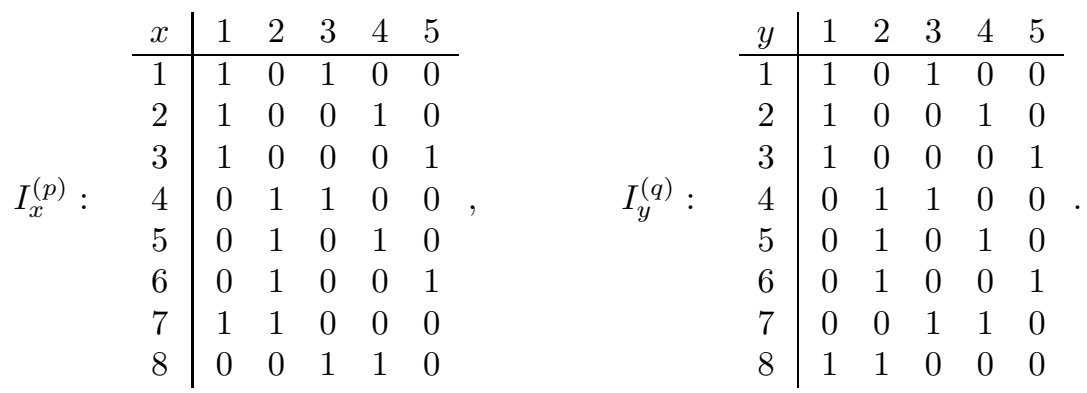

Further computational searches have revealed that $N_{2}(6,2,2,3)=12, N_{2}(6,2,3,5)=13$, $N_{2}(7,2,2,3)=21$ and $N_{2}(7,3,3,8) \geq 26$. The remaining upper bounds given in Table 3 follow from these results.

Finally, returning to the general case, we remark that the lower bounds in Table 2 and upper bounds in Table 3 , give respectively, lower and upper bounds on $\mathcal{P}_{\text {wce }}^{1}\left(n, m_{A}, m_{B}\right)$, if we identify $m=\min \left\{m_{A}, m_{B}\right\}$ in both tables. This follows from the trivial inequality $\mathcal{P}_{\text {wce }}^{1}\left(n, m_{A}, m_{B}-1\right) \geq \mathcal{P}_{\text {wce }}^{1}\left(n, m_{A}, m_{B}\right) \geq \mathcal{P}_{\text {wce }}^{1}\left(n, m_{A}+1, m_{B}\right)$. The inequalities $\mathcal{P}_{\text {wce }}^{1}(n+$ $\left.1, m_{A}, m_{B}\right) \geq \mathcal{P}_{\text {wce }}^{1}\left(n, m_{A}, m_{B}\right) \geq \mathcal{P}_{\text {wce }}^{1}\left(n+1, m_{A}+1, m_{B}+1\right)$ also hold in the general case.

\section{Quantum fingerprinting}

\subsection{One-way model: $m_{B}=n$}

In the quantum fingerprinting scenario, Alice's and Bob's classical fingerprints ( $a$ and $b$ ) and probability distributions $[p(a \mid x)$ and $q(b \mid y)]$ are replaced by quantum states $\rho(x) \in \mathrm{Q}\left(\mathbb{C}^{m_{A}}\right)$ and $\tau(y) \in \mathrm{Q}\left(\mathbb{C}^{m_{B}}\right)$, respectively, where $\mathrm{Q}\left(\mathbb{C}^{d}\right) \equiv\left\{\rho \in \operatorname{End}\left(\mathbb{C}^{d}\right) \mid \rho \geq 0, \operatorname{tr}(\rho)=1\right\}$. Quantum fingerprinting becomes classical when $\rho(x)$ and $\tau(y)$ are diagonal in the computational basis, in which case we may identify $p(a \mid x)=\langle a|\rho(x)| a\rangle$ and $q(b \mid y)=\langle b|\tau(y)| b\rangle$. 
We will first investigate quantum fingerprinting when $m_{B}=n$, in which case Bob encodes Sapna's messages into orthogonal quantum states, upon which Roger may perform an orthogonal measurement to unambiguously determine the state, and thus $y$. Though implemented with quantum states, this portion of the fingerprinting procedure is effectively classical. Alice, however, must choose her fingerprints from a Hilbert space of dimension $m_{A}=m<n$. Roger's task is then to test whether the state received from Alice, $\rho(x)$, is $\rho(y)$ or not, with one-sided error, and thus the projective measurement $\{P(y), 1-P(y)\}$, where $P(y)$ projects onto the support of $\rho(y)$, is the only suitable choice. The worst-case error probability for this strategy is

$$
P_{\mathrm{wce}}=\max _{x \neq y} \operatorname{tr}[\rho(x) P(y)] .
$$

It is intuitively reasonable that Alice should fingerprint with pure states, and thus, we will first consider this case. The worst-case error probability is then simply the maximal pairwise overlap for Alice's choice of fingerprint states $\{|\psi(x)\rangle\}_{x=1}^{n} \subset \mathbb{C}^{m}$ :

$$
P_{\text {wce }}=\max _{x \neq y}|\langle\psi(x) \mid \psi(y)\rangle|^{2} .
$$

Consequently, the minimum possible worst-case error probability will be achieved when Alice chooses her set of fingerprint states to have the minimum possible maximal pairwise overlap. Defining the quantity

$$
\delta^{2}(n, m) \equiv \min _{\left\{\left|\psi_{k}\right\rangle\right\}_{k=1}^{n} \subset \mathbb{C}^{m}} \max _{j \neq k}\left|\left\langle\psi_{j} \mid \psi_{k}\right\rangle\right|^{2},
$$

our goal is to now find sets of $n$ pure quantum states in $\mathbb{C}^{m}$ which achieve the minimum, called optimal Grassmannian packings [24, 25, 26]. We will assume that $n \geq m>1$ in the following.

Lower bounds on $\delta^{2}(n, m)$ follow from an isometric embedding of the space of quantum states into Euclidean space [24, 25]. Each $\rho$ is first associated with a traceless Hermitian matrix under the correspondence $A(\rho) \equiv \sqrt{\frac{m}{m-1}}\left(\rho-\frac{1}{m} I\right)$. Endowed with the Frobenius norm $\|A\|_{F} \equiv \sqrt{\operatorname{tr}\left(A^{\dagger} A\right)}$, the set of all traceless Hermitian matrices forms a real normed vector space of dimension $m^{2}-1$, in which the images of pure states lie on the unit sphere $\|A\|_{F}=1$. Through an appropriate parametrization of $A$, the Frobenius distance for traceless Hermitian matrices corresponds to Euclidean distance in $\mathbb{R}^{m^{2}-1}$. For example, taking $x_{k}(A)=\operatorname{tr}\left(\lambda_{k} A\right)$, where the lambda matrices $\lambda_{k}\left(k=1, \ldots, m^{2}-1\right)$ form a traceless Hermitian operator basis (see Appendix $\mathrm{A}$ of [27]), we find that $\|A-B\|_{F}=\|\mathbf{x}(A)-\mathbf{x}(B)\|_{2}$. Consequently, given that for pure states $\|A(\psi)-A(\phi)\|_{F}^{2}=\frac{2 m}{m-1}\left(1-|\langle\psi \mid \phi\rangle|^{2}\right)$, a bound on the maximum possible minimal distance between $n$ points on the unit sphere in $\mathbb{R}^{m^{2}-1}$ will imply a bound on $\delta^{2}(n, m)$.

Packings of points on the unit sphere in $\mathbb{R}^{d}$ are called spherical codes [28]. The Rankin bounds for spherical codes [29] imply for Grassmannian packings, the simplex bound [24, 25]

$$
\delta^{2}(n, m) \geq \frac{n-m}{m(n-1)},
$$

with equality possible only when $n \leq m^{2}$, and the orthoplex bound

$$
\delta^{2}(n, m) \geq \frac{1}{m},
$$




\begin{tabular}{|c|c|c|c|c|c|c|c|c|c|c|c|c|}
\hline$n \backslash m$ & 2 & 3 & 4 & 5 & 6 & 7 & 8 & 9 & 10 & 11 & 12 & 13 \\
\hline 2 & 0 & 0 & 0 & 0 & 0 & 0 & 0 & 0 & 0 & 0 & 0 & 0 \\
\hline 3 & $1 / 4^{e}$ & 0 & 0 & 0 & 0 & 0 & 0 & 0 & 0 & 0 & 0 & 0 \\
\hline 4 & $1 / 3^{e}$ & $1 / 9^{e}$ & 0 & 0 & 0 & 0 & 0 & 0 & 0 & 0 & 0 & 0 \\
\hline 5 & $1 / 2$ & .1886 & $1 / 16^{e}$ & 0 & 0 & 0 & 0 & 0 & 0 & 0 & 0 & 0 \\
\hline 6 & $\mathbf{1} / \mathbf{2}^{m}$ & $1 / 5^{e}$ & .1071 & $1 / 25^{e}$ & 0 & 0 & 0 & 0 & 0 & 0 & 0 & 0 \\
\hline 7 & .6051 & $2 / 9^{e}$ & $1 / 8^{e}$ & .0709 & $\mathbf{1} / \mathbf{3 6}^{e}$ & 0 & 0 & 0 & 0 & 0 & 0 & 0 \\
\hline 8 & .6306 & $1 / 4$ & $1 / 7^{e}$ & .0871 & .0502 & $1 / 49^{e}$ & 0 & 0 & 0 & 0 & 0 & 0 \\
\hline 9 & $2 / 3$ & $1 / 4^{e}$ & .1615 & .1025 & $1 / 16^{e}$ & .0384 & $1 / 64^{e}$ & 0 & 0 & 0 & 0 & 0 \\
\hline 10 & .7022 & $1 / 3$ & .1687 & $1 / 9^{e}$ & .0741 & .0491 & .0299 & $1 / 81^{e}$ & 0 & 0 & 0 & 0 \\
\hline 11 & .7236 & $1 / 3$ & .1808 & $3 / 25^{e}$ & $1 / 12^{e}$ & .0573 & .0394 & .0238 & $1 / 100^{e}$ & 0 & 0 & 0 \\
\hline 12 & .7236 & $\mathbf{1} / \mathbf{3}^{m}$ & .1830 & .1277 & $1 / 11^{e}$ & .0651 & .0456 & .0319 & .0193 & $1 / 121^{e}$ & 0 & 0 \\
\hline 13 & .7713 & .3871 & $3 / 16^{e}$ & .1351 & .0972 & .0714 & .0522 & $\mathbf{1} / \mathbf{2 7 ^ { e }}$ & .0265 & .0163 & $1 / 144^{e}$ & 0 \\
\hline 14 & .7820 & .4066 & $1 / 5$ & .1411 & .1026 & $1 / 13^{e}$ & .0577 & .0429 & .0310 & .0222 & .0138 & $1 / 169^{e}$ \\
\hline 15 & .7963 & .4141 & $1 / 5$ & .1452 & .1072 & $4 / 49^{e}$ & $1 / 16^{e}$ & .0476 & .0357 & .0262 & .0189 & .0118 \\
\hline 16 & .8061 & .4196 & $1 / 5^{e}$ & .1506 & $1 / 9^{e}$ & .0857 & $1 / 15^{e}$ & .0519 & $1 / 25^{e}$ & .0303 & $1 / 45^{e}$ & .0162 \\
\hline 17 & .8140 & .4282 & $1 / 4$ & .1549 & .1154 & .0893 & .0703 & .0556 & .0438 & .0341 & .0261 & .0196 \\
\hline 18 & .8243 & .4395 & $1 / 4$ & .1581 & .1194 & .0926 & .0735 & $1 / 17^{e}$ & .0471 & .0374 & .0294 & .0227 \\
\hline 19 & .8366 & .4576 & $1 / 4$ & .1581 & .1220 & .0954 & .0764 & $5 / 81^{e}$ & $1 / 20^{e}$ & .0404 & .0324 & .0257 \\
\hline 20 & .8382 & .4712 & $1 / 4^{m}$ & .1581 & .1254 & .0983 & .0790 & .0643 & $1 / 19^{e}$ & .0431 & .0351 & .0284 \\
\hline 21 & .8497 & .4733 & .2890 & $4 / 25^{e}$ & .1271 & .1011 & .0813 & .0667 & .0550 & .0455 & .0375 & .0308 \\
\hline 22 & .8552 & .4948 & .2979 & $1 / 6$ & .1293 & .1039 & .0835 & .0688 & .0571 & .0476 & .0397 & .0330 \\
\hline
\end{tabular}

Table 4. The smallest known maximal pairwise overlap for sets of $n$ pure states in $\mathbb{C}^{m}$. Entries in boldface signify that the overlap is optimal. This is the case for all ETFs (denoted by a superscript e) and MUBs $(m)$. Each maximal overlap is also an achievable worst-case error probability for a quantum fingerprinting strategy under the one-way communication model $\left(m_{B}=n\right)$, with the entries in boldface being the minimum achievable.

if $n>m^{2}$, with equality possible only when $n \leq 2\left(m^{2}-1\right)$.

The simplex bound was derived by Welch [30] and also appears in the context of frame theory $[26,31]$ where a set of states which achieves equality is called an equiangular tight frame (ETF). This name derives from the fact that equality will occur if and only if [26]

$$
\left|\left\langle\psi_{j} \mid \psi_{k}\right\rangle\right|^{2}=\frac{n-m}{m(n-1)}
$$

for all $j \neq k$, which automatically guarantees a tight frame: $\sum_{k}\left|\psi_{k}\right\rangle\left\langle\psi_{k}\right|=\frac{n}{m} I$. Trivial examples of ETFs occur when $n=m$ (basis in $\mathbb{C}^{m}$ ) and $n=m+1$ (vertices of the simplex in $\left.\mathbb{R}^{m}\right)$. Excluding these cases, it is necessary that $n \leq \min \left\{m^{2},(n-m)^{2}\right\}$ for an ETF to exist $[26,31]$. This condition, however, is far from sufficient. In Table 4 we give all known examples of an ETF for small $m$ and $n$. Here we tabulate upper bounds on $\delta^{2}(n, m)$. The entries in Table 4 marked by a superscript $e$ signify cases where an analytical construction of an ETF is known. These derive from quadratic residues [33, 38], conference matrices [32, 26, 31, 36, 38], Hadamard matrices [31, 36], graphs [32, 31, 37], and difference sets [35, 34]. Finally, an ETF is conjectured to exist whenever $n=m^{2}[33,39]$, with analytical constructions for $m \leq 8$ and $m=19[33,39,40,41,42]$.

Given the simplex bound on $\delta^{2}(n, m)$ [Eq. (35)], we know that all ETFs, when they exist, are optimal Grassmannian packings. Sets of mutually unbiased bases (MUBs) [43] also give optimal Grassmannian packings. It is known that when $m$ is a prime power, a maximal set of $m+1$ MUBs exists (i.e. $n=m^{2}+m$ ) with constant overlap of $1 / m$ between elements of different bases, thus saturating the orthoplex bound [Eq. 36]. In Table 4 the MUBs are marked by a superscript $m$. 
When $m=2$, the above embedding maps quantum states surjectively into the unit ball in $\mathbb{R}^{3}$ (realizing the Bloch-sphere representation of a qubit), and our problem is exactly equivalent to finding optimal spherical codes in three dimensions. Collections of putatively optimal spherical codes can be found online [44], and have the maximal overlaps in the first column of Table 4 . Of these, only in the cases $n \leq 14$ has optimality been established (see $[45,46])$. For $n=4,6,8$, and 12, the optimal spherical codes are given by the vertices of a regular tetrahedron, regular octahedron, square antiprism, and regular icosahedron, with maximal overlaps $\delta^{2}=1 / 3,1 / 2,(3+\sqrt{2}) / 7$, and $(1+1 / \sqrt{5}) / 2$, respectively [28]. Except for $n=8$, optimality follows from the Fejes Tóth bound [47]: $\delta^{2}(n, 2) \geq \frac{1}{4} \csc ^{2}\left[\frac{\pi n}{6(n-2)}\right]$.

All other entries in Table 4 are the overlaps for the best packings found in a numerical search, and thus, optimality is not guaranteed. In many cases, however, the given overlap almost saturates the simplex bound and thus must be at least very close to optimal.

When $m_{B}=n$, each of the maximal overlaps in Table 4 is the worst-case error probability for a quantum fingerprinting strategy. Comparing these to the classical error probabilities in Table 2, we see that quantum fingerprinting strategies generally outperform classical strategies by a sizeable margin. Note, for example, that when $n=q(q+1)$, where $q$ is a prime power, there exists a classical constant-weight-code strategy [see Eq. (25)] which requires $m=q^{2}$ fingerprints to obtain a worst-case error probability of $P_{\text {wce }}=1 / q$. This class of classical strategies is optimal for at least $q=2$ and 3. There exist ETFs, however, with the parameters $m=q^{2}$ and $n=q(q+1)+1$ (when $q$ is a prime power) having equiangular overlaps of $1 / q^{3}$ [34]. Consequently, for the same $m$ and $n$, the classical worst-case error probability above may be reduced to $P_{\text {wce }}=1 / q^{3}$, or less, using quantum fingerprints. Taking a different approach, if we instead fix $n=q(q+1)$ and $P_{\text {wce }}=1 / q$, we know that a quantum MUBs strategy exists which achieves this performance using only $m=q$ fingerprints. Finally, the quantum performance gain is far more impressive if we instead fix $m$ and $P_{\text {wce }}$. For example, in the classical case, when $m=4$ we know that to achieve $P_{\text {wce }}=1 / 2$ it is necessary that $n \leq 6$. A numerical search, however, finds that there exists a set of $n 4$-dimensional quantum states with a maximum pairwise overlap of $1 / 2$, or less, for all $n \leq 75$. Thus, quantum fingerprinting strategies can map at least 75 messages into 4 fingerprints while retaining a worst-case error probability $P_{\text {wce }} \leq 1 / 2$, compared to only 6 messages classically.

In the above analysis we have assumed that Alice's fingerprint states are pure. Under this restriction, each boldfaced error probability in Table 4 is the minimum possible. Suppose now that Alice is allowed to choose mixed quantum states for her fingerprints. When $m=2$, it is clear from Eq. (32) that pure states remain the only valid choice if she is to retain $P_{\text {wce }}<1$, since any mixed qubit has support on the entire Hilbert space. Consequently, each boldfaced entry in the first column of Table 4 is the minimum achievable worst-case error probability over all (mixed or pure) quantum fingerprinting strategies. We will conclude this subsection by proving that the remaining boldfaced error probabilities in Table 4 are also the minimum achievable.

Define the quantity

$$
\Delta^{2}(n, m) \equiv \min _{\left\{\rho_{k}\right\}_{k=1}^{n} \subset \mathrm{Q}\left(\mathbb{C}^{m}\right)} \max _{j \neq k} \operatorname{tr}\left(\rho_{j} \rho_{k}\right) .
$$

By considering the choice $\rho_{k}=I / m$ for all $k$, we immediately arrive at the upper bound $\Delta^{2}(n, m) \leq 1 / m$. We will now show that the Rankin lower bounds on the maximum pairwise 
overlap for a set of pure quantum states [Eq.'s (35) and (36)] also apply to general quantum states. First note that

$$
\operatorname{tr}\left(\rho_{j} \rho_{k}\right)=\frac{1}{m}+\frac{m-1}{m} \operatorname{tr}\left[A\left(\rho_{j}\right) A\left(\rho_{k}\right)\right]
$$

under the correspondence $A(\rho) \equiv \sqrt{\frac{m}{m-1}}\left(\rho-\frac{1}{m} I\right)$. In the context of deriving lower bounds on $\Delta^{2}(n, m)$, we need only consider sets of quantum states with $\operatorname{tr}\left[A\left(\rho_{j}\right) A\left(\rho_{k}\right)\right] \leq 0$ for all $j \neq k$, since otherwise the maximum pairwise overlap is greater than $1 / m$, which exceeds the upper bound. Assuming $\operatorname{tr}\left[A\left(\rho_{j}\right) A\left(\rho_{k}\right)\right] \leq 0$, and given that $\|A(\rho)\|_{F} \leq 1$ for any quantum state, we see that

$$
\operatorname{tr}\left[A\left(\rho_{j}\right) A\left(\rho_{k}\right)\right] \geq \operatorname{tr}\left[\tilde{A}\left(\rho_{j}\right) \tilde{A}\left(\rho_{k}\right)\right]=1-\frac{1}{2}\left\|\tilde{A}\left(\rho_{j}\right)-\tilde{A}\left(\rho_{k}\right)\right\|_{F}^{2},
$$

where the rescaled $\tilde{A}\left(\rho_{k}\right) \equiv A\left(\rho_{k}\right) /\left\|A\left(\rho_{k}\right)\right\|_{F}$ satisfy $\left\|\tilde{A}\left(\rho_{k}\right)\right\|_{F}=1$. Thus, under the above isometric embedding into $\left(m^{2}-1\right)$-dimensional Euclidean space, the Rankin bounds on spherical codes [29] imply that

$$
\Delta^{2}(n, m) \geq \frac{n-m}{m(n-1)},
$$

and

$$
\Delta^{2}(n, m)=\frac{1}{m}
$$

if $n>m^{2}$. Equality in the latter follows from the upper bound.

Finally, denoting Alice's fingerprint states by $\rho(x)$, and the support of $\rho(x)$ by $P(x)$, the worst-case error probability

$$
\begin{aligned}
P_{\text {wce }} & =\max _{x \neq y} \operatorname{tr}[\rho(x) P(y)] \\
& \geq \max _{x \neq y} \operatorname{tr}[\rho(x) \rho(y)] \\
& \geq \Delta^{2}(n, m),
\end{aligned}
$$

and consequently, each boldfaced error probability in Table 4 is in fact the minimum achievable worst-case error probability over all (mixed or pure) quantum fingerprinting strategies.

\subsection{Generalizations and the SMP model: $m_{A}=m_{B}$}

Now consider quantum strategies under the general fingerprinting scenario $\left(m_{A}, m_{B} \leq n\right)$. If Alice sends state $\rho(x)$ for message $x$, and Bob sends $\tau(y)$, then, given the one-sided-error constraint, it is straightforward to see that Roger's optimal measurement on the product state $\rho(x) \otimes \tau(y)$ to determine whether $x=y$, is the projective measurement $\left\{P_{1}, P_{0}=1-P_{1}\right\}$, where $P_{1}$ projects onto the support of $\tilde{P}_{1} \equiv \sum_{z} \rho(z) \otimes \tau(z)$. The worst-case error probability is then

$$
P_{\text {wce }}=\max _{x \neq y} \operatorname{tr}\left[\rho(x) \otimes \tau(y) P_{1}\right] .
$$

It remains to find good choices for $\rho(x)$ and $\tau(y)$. For simplicity, we will restrict our study to strategies where the fingerprint states are pure, $\rho(x)=|\psi(x)\rangle\langle\psi(x)|$ and $\tau(y)=|\phi(y)\rangle\langle\phi(y)|$ say. 
Consider the case where $m_{A}=m_{B}=m$, and suppose Alice and Bob map messages onto the same set of fingerprint states, $\{|\psi(x)\rangle\}_{x=1}^{n} \subset \mathbb{C}^{m}$ say. If Roger, instead of choosing to project onto the support of $\sum_{z}|\psi(z)\rangle\langle\psi(z)|\otimes| \psi(z)\rangle\langle\psi(z)|$, fixes $P_{1}=\Pi_{\mathrm{sym}} \equiv \frac{1}{2} \sum_{a, b}|a\rangle\langle a| \otimes$ $|b\rangle\langle b|+| a\rangle\langle b|\otimes| b\rangle\langle a|$, the projector onto the symmetric subspace of $\mathbb{C}^{m} \otimes \mathbb{C}^{m}$, then the resulting worst-case error probability is

$$
P_{\text {wce }}=\max _{x \neq y} \frac{1}{2}\left(1+|\langle\psi(x) \mid \psi(y)\rangle|^{2}\right) .
$$

The optimal Grassmannian packings described in the previous subsection are now the best choices for $\{|\psi(x)\rangle\}_{x=1}^{n}$. Although the minimum possible worst-case error probability is bounded below by one half, $P_{\text {wce }}=\frac{1}{2}\left[1+\delta^{2}(n, m)\right] \geq 1 / 2$, these strategies were found to give the best error rates when $n \gtrsim \frac{1}{2} m(m+1)$, the dimension of the symmetric subspace.

We now describe analytical constructions of quantum fingerprinting strategies from ETFs under the SMP model. For an ETF, the strategy just described has $P_{\mathrm{wce}}=\frac{1}{2}\left[1+\frac{n-m}{m(n-1)}\right]$. However when $n<\frac{1}{2} m(m+1)$ we can obtain smaller worst-case error probabilities in the following way. Suppose the set $\left\{\left|\xi_{j}\right\rangle\right\}_{j=1}^{n}$ forms an ETF in $\mathbb{C}^{m}$. We choose $|\psi(x)\rangle=\left|\xi_{x}\right\rangle$ and $|\phi(y)\rangle=\left|\xi_{y}{ }^{*}\right\rangle$, for Alice's and Bob's fingerprint states respectively, where conjugation is done in the computational basis. Note that $\tilde{P}_{1} \equiv \sum_{z}|\psi(z)\rangle\langle\psi(z)|\otimes| \phi(z)\rangle\langle\phi(z)|$ is the sum of $n$ rank-1 operators, and hence, has rank no greater than $n$. Using the defining property of an ETF [Eq. (37)], it is straightforward to verify that the $n$ states

$$
\begin{aligned}
\left|\Xi_{k}\right\rangle & \equiv \sqrt{\frac{m(n-1)}{n^{2}(m-1)}} \sum_{j=1}^{n} e^{\frac{2 \pi i j k}{n}}\left|\xi_{j}\right\rangle \otimes\left|\xi_{j}^{*}\right\rangle, \quad k=1, \ldots, n-1, \\
\left|\Xi_{n}\right\rangle & \equiv \sqrt{\frac{m}{n^{2}}} \sum_{j=1}^{n}\left|\xi_{j}\right\rangle \otimes\left|\xi_{j}^{*}\right\rangle
\end{aligned}
$$

are orthonormal and belong to the support of $\tilde{P}_{1}$. Consequently, these states span the support of $\tilde{P}_{1}$, and Roger's optimal measurement has $P_{1}=\sum_{k=1}^{n}\left|\Xi_{k}\right\rangle\left\langle\Xi_{k}\right|$. The worst-case error probability for the strategy may now be calculated in a straightforward manner. The result is $P_{\text {wce }}=\frac{n^{2}-m^{2}}{m^{2}(n-1)}$.

When $n=2 m$, ETFs can be used to obtain even smaller worst-case error probabilities. Suppose again that the set $\left\{\left|\xi_{j}\right\rangle\right\}_{j=1}^{n}$ forms an ETF in $\mathbb{C}^{m}$. Define the $m \times n$ matrix with entries $X_{j k} \equiv \sqrt{\frac{m}{n}}\left\langle j \mid \xi_{k}\right\rangle$. The tight frame condition, $\sum_{k}\left|\xi_{k}\right\rangle\left\langle\xi_{k}\right|=\frac{n}{m} I$, implies that the rows of $X$ are orthonormal: $X X^{\dagger}=I$. Thus, another $n-m$ rows may be appended to form an $n \times n$ unitary matrix $U=\left[\frac{X}{Y}\right]$, where $X Y^{\dagger}=Y X^{\dagger}=0$ and $Y Y^{\dagger}=I$. This last relation implies that a tight frame in $\mathbb{C}^{n-m}$ may be constructed from the columns of $Y$ by setting $\left\langle j \mid \chi_{k}\right\rangle=\sqrt{\frac{n}{n-m}} Y_{j k}$. The set $\left\{\left|\chi_{j}\right\rangle\right\}_{j=1}^{n}$ is in fact an ETF. This follows from the unitarity of $U: I=U^{\dagger} U=X^{\dagger} X+Y^{\dagger} Y$, i.e. $\delta_{j k}=\frac{m}{n}\left\langle\xi_{j} \mid \xi_{k}\right\rangle+\frac{n-m}{n}\left\langle\chi_{j} \mid \chi_{k}\right\rangle$. Thus, when $j \neq k$ we have $\left|\left\langle\chi_{j} \mid \chi_{k}\right\rangle\right|^{2}=\frac{m^{2}}{(n-m)^{2}}\left|\left\langle\xi_{j} \mid \xi_{k}\right\rangle\right|^{2}=\frac{m}{(n-m)(n-1)}$, which is the defining property of an ETF in $\mathbb{C}^{n-m}$. We have shown that the existence of an ETF in $\mathbb{C}^{m}$ guarantees the existence of an ETF (of equal size) in $\mathbb{C}^{n-m}$ [31]. Note that for the special case $n=2 m$, the second ETF also belongs to $\mathbb{C}^{m}$. Importantly, again from the unitarity of $U$, the inner products of the ETF pair satisfy $\left\langle\chi_{j} \mid \chi_{k}\right\rangle=-\left\langle\xi_{j} \mid \xi_{k}\right\rangle$ for $j \neq k$. Using this relation and Eq. (37), it is 


\begin{tabular}{|c|ccc|}
\hline$n \backslash m$ & 2 & 3 & 4 \\
\hline 2 & 0 & 0 & 0 \\
3 & $5 / 8^{e}$ & 0 & 0 \\
4 & $2 / 3^{e}$ & $7 / 27^{e}$ & 0 \\
5 & $3 / 4$ & .4330 & $9 / 64^{e}$ \\
6 & $3 / 4^{m}$ & $7 / 15^{e}$ & .2494 \\
7 & .8025 & $11 / 18^{e}$ & .3398 \\
8 & .8153 & $5 / 8$ & $5 / 14^{e}$ \\
9 & $5 / 6$ & $5 / 8^{e}$ & .4962 \\
10 & .8511 & $2 / 3$ & .5567 \\
11 & .8618 & $2 / 3$ & .5904 \\
12 & .8618 & $2 / 3^{m}$ & .5915 \\
13 & .8857 & .6935 & $19 / 32^{e}$ \\
14 & .8910 & .7033 & $3 / 5$ \\
15 & .8982 & .7071 & $3 / 5$ \\
16 & .9031 & .7098 & $3 / 5^{e}$ \\
17 & .9070 & .7141 & $5 / 8$ \\
18 & .9122 & .7198 & $5 / 8$ \\
19 & .9183 & .7288 & $5 / 8$ \\
20 & .9191 & .7356 & $5 / 8^{m}$ \\
21 & .9249 & .7367 & .6445 \\
22 & .9276 & .7474 & .6490 \\
\hline
\end{tabular}

Table 5. The smallest known worst-case error probabilities for a quantum fingerprinting strategy under the SMP model $\left(m_{A}=m_{B}=m\right)$.

straightforward to verify that the $n-1$ states

$$
\left|\Xi_{k}\right\rangle \equiv \frac{\sqrt{n-1}}{n} \sum_{j=1}^{n} e^{\frac{2 \pi i j k}{n}}\left|\xi_{j}\right\rangle \otimes\left|\chi_{j}{ }^{*}\right\rangle, \quad k=1, \ldots, n-1
$$

are orthonormal and belong to the support of $\tilde{P}_{1}$, where we are choosing $|\psi(x)\rangle=\left|\xi_{x}\right\rangle$ and $|\phi(y)\rangle=\left|\chi_{y}{ }^{*}\right\rangle$ for Alice's and Bob's fingerprint states respectively. Unlike in the previous case, $\sum_{j}\left|\xi_{j}\right\rangle \otimes\left|\chi_{j}{ }^{*}\right\rangle=0$, which means $\operatorname{rank} \tilde{P}_{1} \leq n-1$. Consequently, Roger's optimal measurement has $P_{1}=\sum_{k=1}^{n-1}\left|\Xi_{k}\right\rangle\left\langle\Xi_{k}\right|$. The worst-case error probability for this quantum fingerprinting strategy is $P_{\text {wce }}=\frac{3 m-2}{m(2 m-1)}$.

In Table 5, putatively optimal worst-case error probabilities are collected for $m=2,3$ and 4. Strategies which use ETFs or MUBs are marked by a superscript $e$ or $m$, respectively. When $n<\frac{1}{2} m(m+1)$ some of the analytical quantum fingerprinting strategies described above are outperformed by those found numerically, and the corresponding numerical worstcase error probability is given instead. Numerical strategies were located by setting $P_{1}$ to the support of $\tilde{P}_{1} \equiv \sum_{z}|\psi(z)\rangle\langle\psi(z)|\otimes| \phi(z)\rangle\langle\phi(z)|$ as the fingerprint sets $\{|\psi(x)\rangle\}_{x=1}^{n}$ and $\{|\phi(y)\rangle\}_{y=1}^{n}$ were varied across Hilbert space. Although none of the quantum error rates in Table 5 can be claimed optimal, the lower bounds on the classical error rates in Table 3 are still surpassed by a fair margin. Again, the performance gain is most impressive when we consider $m$ and $P_{\text {wce }}$ fixed. For example, in the classical case, when $m=4$ we know that to achieve $P_{\text {wce }}=3 / 4$ it is necessary that $n \leq 6$. There exists a set of $n 4$-dimensional quantum states, however, with a maximum pairwise overlap of $1 / 2$, or less, for all $n \leq 75$. Thus, quantum fingerprinting strategies can map at least 75 messages into 4 fingerprints while retaining $P_{\text {wce }} \leq 3 / 4$ [see Eq. (47)], compared to only 6 messages classically. 


\section{Conclusion}

In this article we have derived lower bounds on the worst-case error probability for a classical fingerprinting protocol with one-sided error which are applicable in the small-message limit. These are our main results and the content of Theorems 4, 7, 10 and 13. Although the majority of the lower bounds are tight only in the one-way communication model, they also apply to a generalized scenario which encompasses the simultaneous message passing model. Furthermore, the set-theoretic techniques used for their derivation might be of interest from an asymptotic point of view. Additionally, we have presented quantum fingerprinting protocols derived from spherical codes, equiangular tight frames and mutually unbiased bases, with error rates surpassing the classical bounds. We hope that our work provides some important new results applicable to current experimental investigations of quantum fingerprinting protocols $[10,11]$. The absolute limits of successful fingerprinting protocols provide quantitative measures for the compressibility of information stored in message strings. Our analysis may be appended to the growing list which reveal a fundamentally greater capacity to compress data stored as quantum information.

\section{Acknowledgments}

AJS would like to thank Harry Buhrman, Nicholas Cavenagh, Dmitry Gavinsky and John Watrous for helpful discussions. This work has been supported by CIAR, CSE, iCORE and MITACS. JW acknowledges support from AIF and PIMS.

\section{References}

1. E. Kushilevitz and N. Nisan, Communication Complexity (Cambridge University Press, Cambridge, 1997).

2. A. C.-C. Yao, "Some complexity questions related to distributive computing," in Proceedings of the 11th Annual ACM Symposium on Theory of Computing, Atlanta, 1979, edited by M. J. Fischer et al. (ACM, New York, 1979), p. 209.

3. A. Ambainis, "Communication complexity in a 3-computer model," Algorithmica 16, 298 (1996).

4. I. Newman and M. Szegedy, "Public vs. private coin flips in one round communication games," in Proceedings of the 28th Annual ACM Symposium on Theory of Computing, Philadelphia, 1996, edited by G. L. Miller (ACM, New York, 1996), p. 561.

5. L. Babai and P. G. Kimmel, "Randomized simultaneous messages: solution of a problem of Yao in communication complexity," in Proceedings of the 12th Annual IEEE Conference on Computational Complexity, Ulm, Germany, 1997, (IEEE Comp. Soc., Los Alamitos CA, 1997), p. 239.

6. H. Buhrman, R. Cleve, J. Watrous, and R. de Wolf, "Quantum fingerprinting," Phys. Rev. Lett. 87, 167902 (2001).

7. A. C.-C. Yao, "On the power of quantum fingerprinting," in Proceedings of the 35th Annual ACM Symposium on Theory of Computing, Atlanta, 1999, edited by J. S. Vitter et al. (ACM, New York, 2003), p. 77.

8. J. Niel de Beaudrap, "One-qubit fingerprinting schemes," Phys. Rev. A 69, 022307 (2004).

9. A. Ambainis and Y. Shi, "Distributed construction of quantum fingerprints," Quantum Inf. Comput. 4, 146 (2004)

10. R. T. Horn, S. A. Babichev, K.-P. Marzlin, A. I. Lvovsky, B. C. Sanders, "Single-qubit optical quantum fingerprinting," Phys. Rev. Lett. 95, 150502 (2005).

11. J. Du, P. Zou, D. K. L. Oi, X. Peng, L. C. Kwek, C. H. Oh, A. Ekert, "Experimental demonstration of quantum state multi-meter and one-qubit fingerprinting in a single quantum device," arXiv:quant-ph/0411180. 
12. R. T. Horn, A. J. Scott, J. Walgate, R. Cleve, A. I. Lvovsky and B. C. Sanders, "Classical and quantum fingerprinting with shared randomness and one-sided error," Quantum Inf. Comput. 5, 258 (2005).

13. I. Anderson, Combinatorics of Finite Sets (Oxford University Press, Oxford, 1987).

14. K. Engel, Sperner Theory (Cambridge University Press, Cambridge, 1997).

15. L. Babai and P. Frankl, Linear Algebra Methods in Combinatorics, unpublished manuscript.

16. E. Sperner, "Ein Satz über Untermengen einer endlichen Menge," Math. Z. 27, 544 (1928).

17. L. Lovász, Combinatorial problems and exercises (North-Holland, Amsterdam, 1979).

18. P. Erdös, P. Frankl and Z. Füredi, "Families of finite sets in which no set is covered by the union of two others," J. Combin. Theory Ser. A 33, 158 (1982).

19. P. Erdös, P. Frankl and Z. Füredi, "Families of finite sets in which no set is covered by the union of $r$ others," Israel J. Math. 33, 79 (1985).

20. W. H. Kautz and R. C. Singleton, "Nonrandom binary superimposed codes," IEEE Trans. Inf. Theory 10, 363 (1964).

21. H. K. Kim and V. Lebedev, "On optimal superimposed codes," J. Combin. Designs 12, 79 (2004).

22. A. E. Brouwer, J. B Shearer, N. J. A. Sloane and W. D. Smith, "A new table of constant weight codes," IEEE Trans. Inf. Theory 36, 1334 (1990).

23. E. Agrell, A. Vardy and K. Zeger, "Upper bounds for constant-weight codes," IEEE Trans. Inf. Theory 46, 2373 (2000).

24. J. H. Conway, R. H. Hardin and N. J. A. Sloane, "Packing lines, planes, etc.: packings in Grassmannian spaces," Experiment. Math. 5, 139 (1996).

25. A. Barg, T. Richardson and R. Urbanke, "Some good multiple-antenna codes," unpublished.

26. T. Strohmer and R. W. Heath Jr., "Grassmannian frames with applications to coding and communication," Appl. Comp. Harm. Anal. 14, 257 (2003).

27. P. Rungta, V. Buzek, C. M. Caves, M. Hillery, and G. J. Milburn, "Universal state inversion and concurrence in arbitrary dimensions," Phys. Rev. A 64, 042315 (2001).

28. J. H. Conway and N. J. A. Sloane, Sphere Packings, Lattices and Groups (Springer-Verlag, New York, 1988).

29. R. A. Rankin, "The closest packing of spherical caps in $n$ dimensions," Proc. Glasgow Math. Assoc. 2, 139 (1955).

30. L. R. Welch, "Lower bounds on the maximum cross correlation of signals," IEEE Trans. Inf. Theory 20, 397 (1974).

31. R. B. Holmes and V. I. Paulsen, "Optimal frames for erasures," Lin. Alg. Appl. 377, 31 (2004).

32. P. Delsarte, J. M. Goethals and J. J. Seidel, "Bounds for systems of lines, and Jacobi polynomials," Philips Res. Rep. 30, 91* (1975).

33. G. Zauner, "Quantendesigns - Grundzüge einer nichtkommutativen Designtheorie," PhD thesis (University of Vienna, 1999).

34. H. König, "Cubature formulas on spheres," in Advances in Multivariate Approximation, edited by W. Haußmann, K. Jetter and M. Reimer, (Wiley-VCH, Berlin, 1999), p. 201.

35. P. Xia, S. Zhou and G. B. Giannakis, "Achieving the Welch bound with difference sets," IEEE Trans. Inf. Theory 51, 1900 (2005).

36. B. G. Bodmann and V. I. Paulsen, "Frames, graphs and erasures," Lin. Alg. Appl. 404, 118 (2005).

37. M. A. Sustik, J. A. Tropp, I. S. Dhillon and R. W. Heath Jr., "On the existence of equiangular tight frames," preprint.

38. J. M. Renes, "Equiangular tight frames from Paley tournaments," arXiv:math.FA/0408287.

39. J. M. Renes, R. Blume-Kohout, A. J. Scott and C. M. Caves, "Symmetric informationally complete quantum measurements," J. Math. Phys. 45, 2171 (2004).

40. S. G. Hoggar, "64 lines from a quaternionic polytope," Geom. Dedic. 69, 287 (1998).

41. M. Grassl, "On SIC-POVMs and MUBs in dimension 6," in Proceedings of the 2004 ERATO Conference on Quantum Information Science, Tokyo, 2004, p. 60.

42. D. M. Appleby, "Symmetric informationally complete-positive operator valued measures and the extended Clifford group," J. Math. Phys. 46, 052107 (2005). 
43. W. K. Wootters and B. D. Fields, "Optimal state-determination by mutually unbiased measurements," Ann. Phys. 191, 363 (1989).

44. N. J. A. Sloane et al., Spherical Codes, published electronically at http://www.research.att.com/ $\sim$ njas/packings/

45. D. A. Kottwitz, "The densest packing of equal circles on a sphere," Acta Cryst. A 47, 158 (1991).

46. J. Buddenhagen and D. A. Kottwitz, "Multiplicity and symmetry breaking in (conjectured) densest packings of congruent circles on a sphere," preprint.

47. L. Fejes Tóth, "The isepiphan problem for n-hedra," Amer. J. Math. 70, 174 (1948). 